\section{P398 DEVELOPMENT OF VIRTUAL REALITY TRAINING CURRICULUM FOR ERCP}

1,2,3Yousef Khouli*, ${ }^{4} J a d$ Alkhoury, 2,3Karim Belhaj, ${ }^{2,3}$ Bijendra Patel. 'Mid Essex Hospitals Nhs Trust, Chelmsford, UK; ${ }^{2}$ Barts Cancer Institute, London, UK; ${ }^{3}$ Queen Mary University of London, London, UK; ${ }^{4}$ Shrewsberry and Telford Hospitals NHS Trust, Telford, UK

10.1136/gutjnl-2020-bsgcampus.472

Introduction Assessment of Endoscopic skills involved in performing Endoscopic Retrograde Cholangiopancreatography (ERCP) in procedural environment is complex. Tutors and experts emphasize the need to develop and use precise and significant assessments measuring tools that are valid for evaluating trainees' progress in obtaining essential gastroenterologyrelated procedural skills. The purpose of this research was to develop a structured evidence-based virtual reality training curriculum and set a proficiency performance benchmark for a set of objective metrics of endoscopic skills during ERCP procedures using the Simbionix GI Mentor 2 Simulator. Our second aim of the study was to form a face and construct validity of the simulator for the ERCP module to show significant differences between competent and non-competent operators in that module.

Materials and Methods In this study, a total of 39 participants were divided into three groups according to their level of experience and number of endoscopies performed in their career (Novices, Intermediate, and Experts). They were required to perform ERCP procedure, case number 2 in Module 1 in the GI Mentor 2 Simulator, and upon completion of the study, they were asked to fill a questionnaire about the simulator, simulation in general, and their previous experience. The time taken for task completion, number of papillary contacts before cannulation, number of cannulation to the Pancreatic Duct, and other metrics calculated from the simulator along with the questionnaire results were collected and compared between the groups. The first group consisted of novices; medical students, foundation doctors, and core trainees, with no previous knowledge or exposure to ERCP or endoscopic procedures.

The second group were categorized as intermediates; specialty trainees from all levels, whom had some experience with endoscopy and has done or assisted in at least 250 procedures. The third group was the expert group, which consisted of consultants in Gastroenterology specialty and each one of them at least has done 2500 procedures in order to be eligible to be placed in the group.

Results We have analysed the data of the participants performance collected from the simulator and compared the results of the three groups together. It was clear that the expert group have done better with shorter time than other groups (264.4 sec); intermediates (321.14 sec), and novices $(822.05 \mathrm{sec})$. The results were analysed further using the IBM SPSS ${ }^{\circledR}$ Software. The date generated showed a statistical significance between the groups having a $\mathrm{p}$ value of $(\mathrm{P}<$ 0.022). Then the experts' results were isolated to define a set of benchmark ranges for the ERCP Module. The average of experts' performance was collected, then we have calculated the Standard Deviation of each mean. Later, the mean of each task was trimmed by excluding any consultant performance beyond the standard deviation by $1 \pm$. six out of twelve metrices were considered significant based on literature ad up do date practice which were included; total time of procedure (178.2 - 361.8 seconds), papilla contact before cannulation (2.25 - 3.25 times), number of cannulations to the PD (1 time), number of cannulations to the CBD (1 time), number of contrast injections to the PD (9.6 - 19.6), and number of contrast injections to the CBD (6.6 - 18.4). The new recalculated mean was used to set a reference criterion and a benchmark range for the performance of the ERCP module in the GI Mentor 2 Simulator.

Afterwards, we recruited these results as a reference in our syllabus. Through analysis of operators' performance and psychological dynamics in practice, we created an evidence-based curriculum that we deemed to be suitable for training ERCP using virtual reality simulator and demonstrated that is possible to define and develop a virtual reality training curriculum for ERCP using structured scientific methodology.

Conclusion The ERCP module in the Simbionix GI Mentor 2 simulator demonstrate face and construct validity as they show statistically significant differences between novice, intermediate, and expert groups as proved in our results and has been done previously in other studies.

We have defined a reference criterion level to develop proficiency performance benchmark for all metrics obtained from our studies on the ERCP procedure based on 5 experts. And we have demonstrated and managed to set a proficiency performance benchmark range in the ERCP module to be used as a baseline when comparing any operator performance on the simulator. Also, our study further our understanding and knowledge of endoscopic expertise and provides trainees with predefined proficiency performance benchmarks that can be used to help and support in their learning of endoscopic skills.

Furthermore, this study has demonstrated that it is possible to define and develop a virtual reality training curriculum for ERCP using structured scientific methodology.

\section{P399 TURNING OUT OF PROGRAM RESEARCH (OOPR) EXPERIENCES INTO S'OOPR EXPERIENCES!}

${ }^{1,2}$ Joseph Tang ${ }^{*},{ }^{1,2} I \mathrm{mran}$ Patanwala. 'Liverpool University Hospitals NHS Foundation Trust, Liverpool, UK; ${ }^{2}$ University of Liverpool, Liverpool, UK

\subsection{6/gutjnl-2020-bsgcampus.473}

Introduction A significant proportion of trainees are interested in an out of program research $(\mathrm{OOPR})^{1}$ to enhance their training and career prospects. $70 \%$ are discouraged from applying due to financial costs. ${ }^{2}$ High quality ward and outpatient based undergraduate training programs are challenging to design and deliver within the day to day working of gastroenterologists.

Methods Our aim was to assess the impact of offering an out of program research trainee a uniquely designed role (education fellow) for one day a week to organise and deliver an additionally funded high quality undergraduate education program in our directorate. This offered an opportunity to improve the financial offerings to the trainee whilst improving and performance managing our training program. The latter was to be delivered through collaboration with the undergraduate education centre, individualising student timetables, formalising induction, organising supervised teaching events and integrating educational governance into the program.

Results An education fellow role was funded via a business case for improving education in 2017 and was found to be attractive to a higher specialist trainee currently on an OOPR. Impact on the conduct of the main research project was felt 